\title{
BRAIN TUMOUR SEGMENTATION STRATEGIES UTILIZING MEAN SHIFT CLUSTERING AND CONTENT BASED ACTIVE CONTOUR SEGMENTATION
}

\author{
D.M. Mahalakshmi and S. Sumathi \\ Department of Electrical Engineering, PSG College of Technology, India
}

\begin{abstract}
This paper proposes an automatic brain tumor segmentation using Mean shift clustering and content based active contour segmentation. In diagnosis of the disease medical imaging has more advantages. Many people suffer from brain tumor, it is a serious and dangerous disease. A brain tumor occurs when abnormal cells form in the brain. A proper diagnosis of brain tumor is provided by the medical imaging. The detection of tumor from brain is an important and difficult task in the medical field. One essential part in detecting the tumor is image segmentation. The brain tumor detection technique in the MRI images is very significant in many symptomatic and cure applications. In view of high amount information in MRI pictures, tumor segmentation and classification are hard. The image segmentation is performed on different dataset of MRI cerebrum tumor pictures. The segmentation gives an automatic brain tumor recognition method to build the exactness, yields with decline in the analysis time. The image segmentation technique includes image acquisition, image preprocessing, denoising, and finally the feature extraction. The input image is pre-processed using wiener filtering and the noise is removed using Edge Adaptive Total Variation Denoising (EATVD) technique. Once the noise is removed from the image, it undergoes segmentation process, where Mean Shift Clustering and Content based active segmentation techniques are used. Finally, the features are extracted from the segmented image using gray level co-occurrence matrix (GLCM). The image segmentation is implemented using MATLAB software. Finally, the tumor is segmented and energy, contrast, correlation, homogeneity is extracted, and comparison results are analyzed.
\end{abstract}

\section{Keywords:}

Edge Adaptive Total Variation Denoising (EATVD), Gray Level Cooccurrence Matrix (GLCM), Magnetic Resonance Imaging (MRI), Convolution Neural Network (CNN), Artificial Neural Network (ANN), Support Vector Machine (SVM)

\section{INTRODUCTION}

In the advanced imaging technology, diagnostic imaging has become an indispensable tool in medicine today. X-ray angiography (XRA), magnetic resonance angiography (MRA), magnetic resonance imaging (MRI), computed tomography (CT) and other imaging modalities are heavily used in clinical practice. Such images provide complementary information about the patient. Due to increase in the complexity in the medical field it requires latest advances in computer technology in order to reduce the cost and it would be possible to develop such automations [1]. Some of the ways for diagnosing brain tumor are MRI scan, CT scan and biopsy of the head etc. In the magnetic imaging techniques and the radio waves are utilized to locate and obtain a digital image of tissues present in the brain. The conventional technique for the MRI is really an exceptionally troubling undertaking and also tedious not to be able to withstand that the expected results are also dependent on the master's experience. Similarly, inspiring a strategy to furnish data to specialists with a precise and furthermore completely programmed system is weighty.

Nowadays brain tumor is a common disease found in the human body. The manual analysis of such images requires training and experience and will often lead to wrong diagnostics. A tumor occurs when abnormal cells form within the brain. The growth of the tumor occupies space within the skull and affects with normal brain activity [3]. Brain tumor is classified into two types as normal brain and abnormal brain and they are termed as malignant or cancerous tumor and benign tumors. Cancerous tumor can be divided into primary tumor that start within the brain and secondary tumor that have spread from somewhere else, known as brain metastasis tumor. Brain tumor detection on medical images forms an essential step in solving several practical applications such as diagnosis of the tumor and registration of patient images obtained at different times. In image processing it is usually necessary to perform high degree of noise reduction in an image before performing higher-level processing steps, such as edge detection [3]. Smoothing filters is used to remove noise from an image. Each pixel is represented by three scalar values representing the red, green and blue chromatic intensities. At each pixel studied, a smoothing filter considers the surrounding pixels to design an accurate version of this pixel. By taking neighboring pixels into considerations, extreme 'noisy' pixels can be represented.

Tumor segmentation algorithms are the key components of automated radiological diagnostic systems. Segmentation methods vary depending on the imaging modality, application domain, method being automatic or semi-automatic and other special factors. Specific segmentation method is not found to extract vasculature from every medical image modality. While techniques like clustering, segmentation etc. employ pure intensity-based pattern recognition techniques such as thresholding followed by connected component analysis, edge detection methods are applied explicitly in the tumor models to extract the tumor contours [2]. Based on the image quality different pre-processing techniques are used in the image segmentation process. On the other hand, methods like low pass filtering, unsharp masking techniques are applied in the postprocessing to overcome the problems arising over segmentation.

In this paper, mean shift clustering algorithm and content based active contour methods are utilized for segmenting the tumor from the brain. The segmentation of the tumor must undergo certain stages. Initially the data is read and pre-processed, then followed by denoising technique. Denoising plays an important role in segmentation, in order to remove the noise from image so that image resolution can be increased. Then it is followed by the segmentation and feature extraction process, where the feature that have similar properties are grouped together and used for classification. 


\section{RELATED WORKS}

The Image segmentation is the process of partitioning a digital image into multiple segments. The goal of segmentation is to simplify or change the representation of an image into the more meaningful information which is easier to analyze. Segmentation plays an important role in analysis of medical images for computer-aided diagnosis and therapy. Segmentation is the challenging and complex task in medical imaging due to imprecise nature of images [1]. For neurological pathology clinical study and research is fully based on the automatic brain tissue classification from magnetic resonance images (MRI) plays a vital role. The important task is to segment the MR images into different classes especially gray matter (GM), white matter (WM) and cerebrospinal fluid (CSF) accurately. The useful diagnostic information can be known from regional volume calculation. In neurodegenerative disorders such as Alzheimer disease, in movement disorders such as Parkinson or Parkinson related syndrome, in white matter metabolic or inflammatory disease, in congenital brain malformations or perinatal brain damage, or in post-traumatic syndrome, quantization of gray and white matter volumes is considered for calculations. The preliminary preprocessing has been completed with the assist of median filter and Ostu's thresholding adopted for threshold determination. Now the preprocessed input MRI is segmented by the usage of K-means clustering algorithm. The key features are extracted from segmented MRI image. They are GLCM, Gabour wavelet and DWT. Since the feature vector received is a massive vector, it is decreased with the assist of Principal Component Analysis (PCA) [18].

A multi stage approach using random forest for brain tumor classification and segmenting them using fast bounding box was proposed [4]. The combined approach of gray level co-occurrence matrix (GLCM) and gray level run length matrix (GLRLM) texture feature extraction methodologies used in this work for classifying the tumor [8]. In this proposed system a comparison is done for classifying the accuracy for the dataset which contains 120 cases of MRI images. The results show that multi stage approach outperforms the reference methods and achieves better accuracy in classifying glioma or meningioma and segmenting the tumor. An important observation in this work is that multi stage approach uses hierarchical classification method which boosts performance significantly. Decisions obtained by applying a hierarchical classification for every single classification task is better than the individual classifying algorithm [8]

The processing is done on the MRI images for the detection and classification of brain tumors that are affected by brain tumors. Pre-processing, segmentation, morphology and so on, are the techniques used to detect the tumor. For extracting features from the MRI image texture feature extraction strategy is utilized [7]. Features are extracted using Gray Level Co-occurrence Matrix. Back propagation neural network (BPNN) and k-nearest neighbor (KNN) Classifier is used after feature extraction for the classification of brain into normal and abnormal images. The neuro-fuzzy classifier used in the system overcomes the inconvenience of artificial neural networks and fuzzy logic that integrates the advantages of two different techniques [17].

An intellectual classification system to recognize normal and abnormal MRI brain images was proposed, where it uses different machine learning techniques: Support Vector Machine (SVM), KNearest Neighbor (KNN) and Hybrid classifier (SVM-KNN) is used to classify images [10]. It is observed from the results that the hybrid classifier SVM-KNN demonstrated the highest classification accuracy rate of $98 \%$ among others. Experimental outcome shows the effectiveness of the SVM and SVM-KNN models. SVM with Quadratic kernel achieves maximum of $96 \%$ classification accuracy and Hybrid classifier (SVM-KNN) achieves $98 \%$ classification accuracy rate on the same test set. This article describes the classification of brain cancer using artificial neural networks for extraction of features [9]. First, MRI database, including Astrocytoma type of brain cancer classified into four types of tumors, namely pilocytic, low grade, anaplastic and multiform glioblastoma, is introduced. Lastly, the artificial neural network architecture is developed to classify brain cancer [16].

A novel algorithm for brain tumor classification is developed using wavelet and Neural Network [11]. This new method is a combination of Discrete Wavelet Transform and Probabilistic Neural Network along with the implementation of GLCM. Using these algorithms, an efficient brain tumor classification method is contracted using the proposed method for optimum extraction of features and efficient brain tumor classification with maximum recognition rate simulation results. The ability of the proposed brain tumor classification method is demonstrated based on obtained results on brain tumor image sets and some combination are taken for training and testing the samples. This system segmented an image using the k-clustering algorithm to generate the initial centroid using a subtractive cluster. At the same time, partial contrast stretching is used to improve the quality of the original image and the median filter is used to improve the segmented image [15].

The system demonstrates an effective method for brain tumor segmentation from 2D MRI images using thresholding technique. The segmentation is also carried out while the presence of the tumor is detected and find the center of the tumor and the tumor perimeter [12]. Morphological operators such as erosion, dilation, also used in the segmented image to remove additional artifacts. By using histogram and global thresholding technique, we successfully separated the tumor region from the MRI background [14]. Brain tumor segmentation based on local binary pattern (LBP) pre-processing and threshold and morphological operations. Most existing methods focus mainly on the detection of the brain tumor. In this context, an eliminate illumination effects using local binary patterns to obtain the exact location of the tumor. False tumor detection can lead to the collapse of patient health. The tumor location has an impact on the normal functioning of human beings. Finally, morphological operations were applied to the LBP image that occurred to obtain a segmented image [13].

\section{PROPOSED SYSTEM}

This paper elaborates on the effectiveness of mean shift clustering and content based active segmentation algorithms in brain tumor segmentation task. The proposed system is analyzed for MRI brain tumor images that are residents and fellows of MRI Research at Post Graduate Institute of Medical Education and Research [2]. The dataset includes 10 variant tumor cases along with 50 image sets. The three types of MRI images mentioned as 
T1 weighted images, T2 weighted images and FLAIR images. This system uses a T2 image set for analyzing. The brain tumor detection consists of various stages for segmenting the tumor from the MR images. The MRI images are initially taken as the input and are pre-processed with a wiener filter. The wiener filter would remove the noise present in the image, and it would blur the image. The pre-processing stage is followed by Denoising, where Edge Adaptive Total Variation technique is used. The main objective of the denoising is to eliminate the unwanted signal present in the input image. The denoised image is further taken to the Segmentation process in which Mean Shift Clustering and content based active contour algorithms are used to segment the pixel that are of similar properties. Finally, the clustered output is used for extracting the features which is done in Feature Extraction phase and the extracted features are used for classifying whether the tumor is present or not. The overview of the proposed system is given in Fig.1.

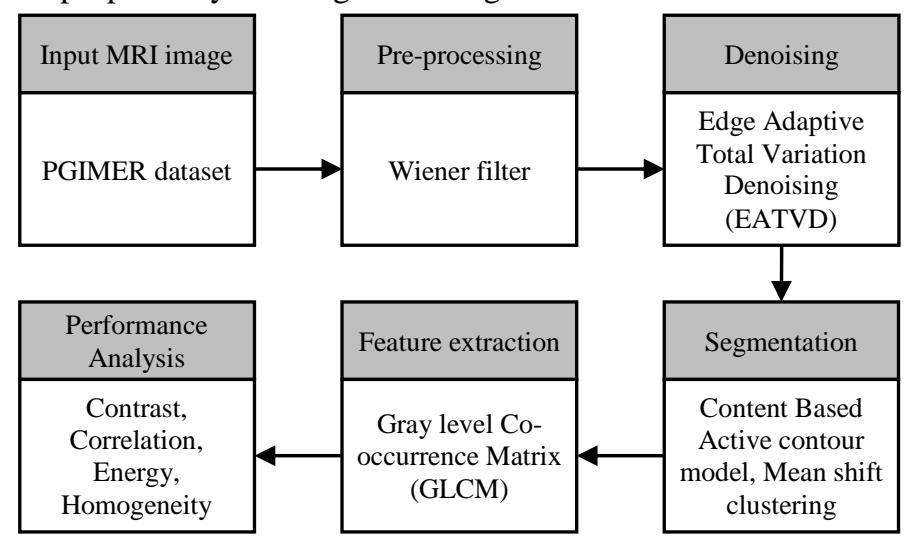

Fig.1. Overview of the proposed system

\subsection{PRE-PROCESSING}

Pre-processing is a common name for image operations at the lowest abstraction level-both input and output are intensity images. The aim of the pre-processing is to improve the image data that suppresses unwanted distortion or improves certain image features important for further processing. The different categories of image pre-processing methods depending on the size of the pixel neighborhood used to calculate the new pixel brightness are as follows.

1. Transformations of pixel brightness.

2. Transformations of geometry.

3. Pre-processing methods that use the processed pixel's local neighborhood.

4. Image recovery requiring knowledge of the entire image.

5. Other pre-processing image classifications exist.

The images obtained from various MRI modalities are influenced by ancient rarities, for example, movement and field inhomogeneity. A MRI can be utilized to assess cerebrum, neck, and spinal string issues. It can likewise enable parental figures to take a gander at issues with your chest, heart, mid-region, joints, or veins. MR image can be procured with a few unique strategies and securing parameters bringing about various image differentiate. The three image types that referenced have a place with the most widely recognized MR acquisitions.MR images can be acquired with different image contrast techniques and acquisition parameters. The three types of images mentioned are among the most common acquisitions of MR [2].

\subsection{WEINER FILTER}

Inverse filtering is a restoration technique for deconvolution that is, when the image is blurred by a known low pass filter, it is possible to recover the image by inverse filtering or generalized inverse filtering. However, inverse filtering is very sensitive to additive noise. The approach of reducing one degradation at a time allows us to develop a restoration algorithm for each type of degradation and simply combine them. The Wiener filtering executes an optimal trade-off between inverse filtering and noise smoothing. It removes the additive noise and inverts the blurring simultaneously. The quality of the filtered image varies with respect to the window size as $3 \times 3,5 \times 5,7 \times 7,13 \times 13$ and $23 \times 23$ and so on.

\subsection{DENOISING}

Digital images play an important role in research and technology, such as geographical information systems, and they play a key role in medical science, such as ultrasound imaging, radiography, computer tomography and MRI. A very large part of digital image processing involves the restoration of images. Image restoration is a method of degradation removal or reduction that occurs during image capture. Degradation is caused by sound and blurring due to electronic and photometric sources. Blurring is the form of bandwidth reduction of images caused by an imperfect process of image formation, such as relative movement between the camera and the original scene or an optical system out of focus. Noise is the undesirable signal that interferes with the original signal and degrades digital image visual quality. Imperfect instruments, problems with data acquisition processes, natural interference, transmission and compression are the main sources of noise in digital images. Image denoising forms the preprocessing step in photography, research, technology and medical science, where the image has somehow been degraded and must be restored before further processing. Edge Adaptive Total Variation Denoising is one among the noise removal techniques which is used in this proposed system.

\subsection{SEGMENTATION}

Segmentation partitions an image in different regions that contain similar attributes for each pixel. The regions should be strongly linked to described objects or features of interest, which are meaningful and useful for image analysis and interpretation. The segmentation is the first step from low - level image processing to convert a gray or color image into one or more other images to high - level image description in terms of features, objects and scenes. The success of the image analysis depends on the reliability of the segmentation, but an accurate image partitioning is usually a very difficult problem. The techniques of segmentation are either contextual or non-contextual thresholds. In this proposed system two different types of segmentation techniques such as Mean Shift Clustering and Content based Active Contour model are used for the analysis and results are compared. 


\subsubsection{Mean Shift Clustering:}

A mean shift is a non-parametric clustering technique used for cluster analysis in computer vision, image processing and for clustering an $n$-dimensional data set. First defining spherical window of radius $r$ in data points and calculate the mean of points which located within the window. Second, the spherical window moves to the next means and repeats until convergence. At each iteration, the spherical window will move dense portion of data set until maximum peak is reached.

\subsubsection{Content based Active Contour Segmentation:}

The active contour technique is a numerical and hypothetical device which is first presented by Osher and Sethian, has turned into a more mainstream hypothetical and numerical structure inside picture preparing, liquid mechanics, designs, computer vision, and so on [5]. In picture segmentation, the active contour strategy has a few favorable circumstances contrasted with the active contour method. The active shape strategy overcomes the troubles of topological changes [19]. In this calculation proposed work has following advances.

1. First produced arbitrary network has same measurement as of input picture at that point join this framework in the picture. Here this assistance in producing the contour in the picture. Contour was at that point show in the picture however this assistance in creating more powerful way.

2. Now find contour position in the image and generate contours which help in finding segmentation of the image. This create initial segmentation for the image.

3. Once these contours were found in the image next is to update the different segment by finding the nearby distance from the segment region. Here if distance is negative then value of the pixel or position of that pixel is considered as part of the segment. Here if the distance is positive then value of the pixel or position of that pixel is taken as outside of the segment.

4. Now next step is to update the segmented area by analyzing the hereby pixel values of the segment. Here function is to be taken so that change in the region can be easily acceptable for any new shape. Active contour model moves in such a way that can merge or split the segments, as per update values [20].

\subsection{FEATURE EXTRACTION}

The component extraction includes improving the measure of assets required to depict an extensive arrangement of information precisely. When performing examination of complex information one of the real issues originates from the quantity of factors included. Examination with a substantial number of factors for the most part requires a lot of memory and calculation control or an order calculation which over fits the preparation test and sums up inadequately to new examples. This is a strategy for catching visual substance of pictures for ordering and recovery. Crude or low dimension picture highlights can be either broad highlights, for example, extraction of shading, surface and shape or area explicit highlights. In this proposed methodology gray scale cooccurrence matrix (GLCM) algorithm is used in-order to extract the true surface feature for the advancement in the estimation of image. The four highlights specifically, Energy, Contrast, Correlation and Homogeneity are figured utilizing MATLAB.
The Gray Level Co-Occurrence Matrix (GLCM) has ended up being a well-known factual technique for removing textural highlight from pictures [6]. As indicated by co-event network, Haralick characterizes fourteen textural highlights estimated from the likelihood lattice to remove the qualities of surface insights of remote detecting pictures. In the proposed technique four critical highlights, Angular Second Moment, Contrast, Correlation and Homogeneity are chosen for usage.

\subsection{SIMULATION RESULTS}

The MRI image dataset that is obtained from Post Graduate Institute of Medical Education and Research (PGIMER) which consists of dataset about 10 cases with 50 image sets in which 10 cases are used for the testing and the results are analyzed in this paper. In Fig. 2 a normal brain tumor MRI image is given as the input to MATLAB. The main aim of the imaging enhancement technique is to process an image So that the resulting image is more suitable than the original for a particular application. Most of enhancement techniques are very problematic and therefore enhancement for one application may be degradation for the other. The acquired input image is then pre-processed using wiener filter by introducing the noise which would result as noisy image.

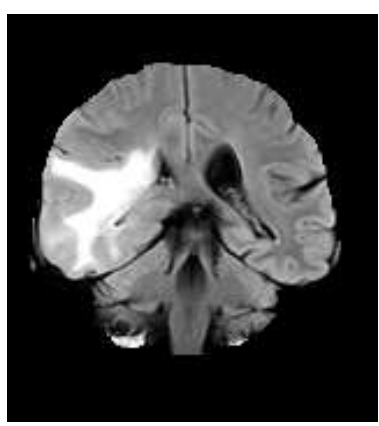

Fig.2. Input original image

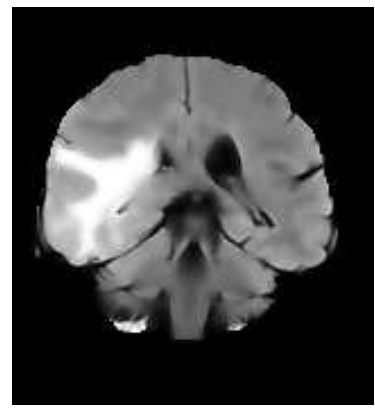

Fig.3. Pre-processed output

In the pre-processing stage very, few amounts of noise are removed, and it is blurred using wiener filter, as shown in Fig.3. The process of the wiener filter is analyzed by varying the window size of the filter as $3 \times 3,5 \times 5,7 \times 7,13 \times 13,15 \times 15$ and $23 \times 23$. When the size of the window is high then the resolution would be minimum while restoring the image. Hence $3 \times 3$ window size taken in the pre-processing stage. 


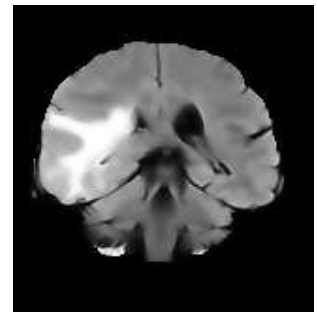

Fig.4. EATVD: Output of the denoising stage

The second process is denoising followed by pre-processing, where EATVD technique is used to eliminate the noise content in the image. The result is shown in the Fig.4. The denoised output is used to calculate the peak signal to noise ratio (PSNR) value, signal to noise ratio (SNR) value, structural similarity (SSIM) value and mean squared error (MSE) value. Although a higher PSNR generally indicates that the reconstruction is of higher quality. Higher the SNR value means more amount of noise is removed. However, as a least squares results, it is slightly biased towards blur. SSIM has been developed to have a quality reconstruction metric that also takes into account the similarity of the edges (high frequency content) between the denoised image and the input image. These values are compared by varying the window size of the filter as $3 \times 3,5 \times 5,7 \times 7,13 \times 13,15 \times 15,23 \times 23$ etc. The comparison results are shown in the Table. 2 and specific value that are computed is given in the Fig.5.

\begin{tabular}{l} 
The Peak-SNR value is 15.1173 \\
The SNR value is 24.1708 \\
The MSE value is 72.7245 \\
The SSIM value is 0.9180 \\
\hline
\end{tabular}

Fig.5. Parameters that are measured from the denoised image

The segmentation is analyzed using two methods, one is Mean Shift Clustering (MSC) algorithm and the other is, Content based active contour model. The outcome of the MSC algorithm returns the average mean shift value as shown in the Fig.5. When the value of the average mean shift is high then the segmentation would have more coverage, which is given in the Fig.6.

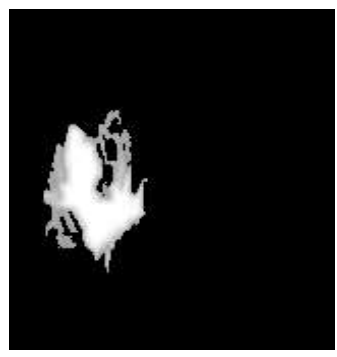

Fig.6. Segmentation result: Content based Active Contour model

The segmentation is also based on the number of iterations that must undergo for segmenting the tumor. By default, the iteration is taken as 100 . When the number of iterations increases more than the limit it may leads to loss in the information. The iteration is plotted in Fig. 7 and it displays the average mean shift value.
Table.1. Segmentation Results of Content Based Active Contour Techniques for Different Images

\begin{tabular}{|c|c|c|c|c|c|}
\hline $\begin{array}{l}\text { Input } \\
\text { image }\end{array}$ & $\begin{array}{l}\text { PSNR } \\
\text { value }\end{array}$ & $\begin{array}{l}\text { SNR } \\
\text { value }\end{array}$ & $\begin{array}{l}\text { MSE } \\
\text { value }\end{array}$ & $\begin{array}{l}\text { SSIM } \\
\text { value }\end{array}$ & $\begin{array}{l}\text { Segmented } \\
\text { image }\end{array}$ \\
\hline & 27.9904 & 19.4678 & 73.3419 & 0.7312 & \\
\hline & 27.7793 & 19.3299 & 73.7799 & 0.7280 & \\
\hline & 27.9733 & 19.4629 & 73.4618 & 0.7309 & \\
\hline & 27.8669 & 19.3334 & 72.9535 & 0.7250 & \\
\hline & 28.3131 & 19.4682 & 70.7316 & 0.7353 & \\
\hline & 27.1193 & 19.6638 & 79.3131 & 0.6417 & \\
\hline & 28.4322 & 19.5464 & 19.5464 & 0.7412 & \\
\hline
\end{tabular}

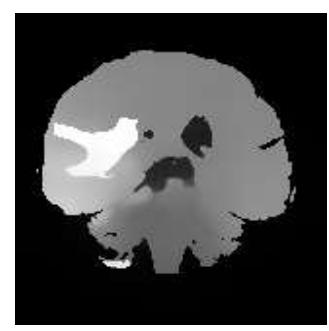

Fig.7. Segmentation result: Mean Shift Clustering

The Table. 1 shows the overall result analysis of various input images and their segmented images. For the given input image, the average mean shift value is maximum as the number of iterations increases as shown in the Fig.8. Finally, the Energy, Contrast, Correlation, Homogeneity features are extracted from the segmented results.

In this proposed system two different cases are taken for extracting the features and the results are shown in the Fig.9 and Fig.10. Finally, the Energy, Contrast, Correlation, Homogeneity features are extracted from the segmented results. In this proposed system different cases are taken for extracting the features. 
Iteration times $=26$;

Averaged Mean - shift $=0.0091619$
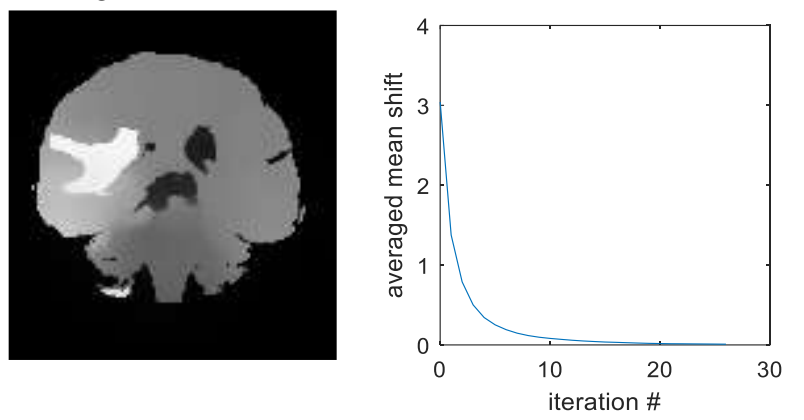

Fig.8. Number of iteration vs average mean shift

The edge adaptive Total Denoising technique is used to preserve the edges in the process of denoising the image. The window size used in the pre-processing phase is $5 \times 5$ to improve the resolution and to remove minimum noise in the image. The EATDV Algorithm, in which SSIM values are calculated, is used in the denoising phase. The PSNR value achieved is the same case by comparing the results obtained with the existing model. Similarly, the time required to calculate the system proposed is found to be relatively short. The denoising is followed by segmentation and feature extraction, where mean shift clustering algorithm and content based active contour techniques are used for segmenting the tumor from the MRI image. The denoising is followed by segmentation and feature extraction, where mean shift clustering algorithm and content based active contour techniques are used for segmenting the tumor from the MRI image.

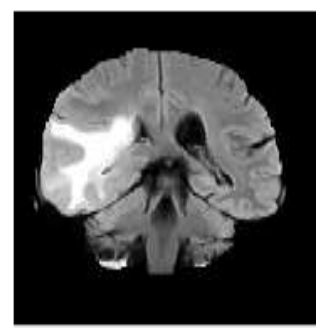

Stats $=$

Struct with fields:

Contrast: [0.9223 0.8000]

Correlation: [0.9570 0.9627]

Correlation: [0.5436 0.5458]

Correlation: [0.9835 0.9857]

Original Image

Fig.9. Feature extraction for image set 1

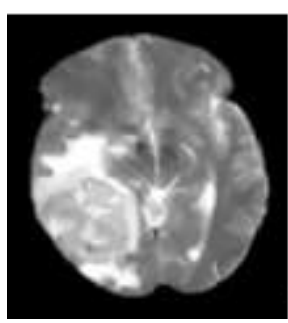

Stats $=$

Struct with fields:

Contrast: [1.1300 0.7524]

Correlation: [0.9506 0.9672]

Correlation: [0.5110 0.5167]

Correlation: [0.9798 0.9866]

Original Image

Fig.10. Feature extraction for image set 2

Finally, features are extracted using GLCM feature extraction techniques in which the texture of the segments describes the Energy, Correlation, Contrast, Homogeneity features that would be used in classification phase. The results for the sample image set in which the denoising parameter is modified are shown in Table.2. Generally, when the PSNR value is high, the image resolution quality is also maximum. In this case, the PSNR value is maximum for the $3 \times 3$ window size, indicating that the image quality is high. If the higher PSNR means that more noise is eliminated, and the texture has a good result. The PSNR value for the $23 \times 23$ window size is the least compared to the $3 \times 3$ window size. In this case, it removes not only more noise, but also part of the texture with a low score.

Table.2. Comparing the Results of Denoised Images

\begin{tabular}{|c|c|c|c|c|}
\hline $\begin{array}{c}\text { Window } \\
\text { Size }\end{array}$ & $\begin{array}{c}\text { Peak-SNR } \\
\text { value }\end{array}$ & $\begin{array}{c}\text { SNR } \\
\text { value }\end{array}$ & $\begin{array}{c}\text { MSE } \\
\text { value }\end{array}$ & $\begin{array}{c}\text { Denoised } \\
\text { image }\end{array}$ \\
\hline $3 \times 3$ & 27.7697 & 8.8576 & 72.2942 & \\
\hline $5 \times 5$ & 25.9420 & 16.9580 & 72.1230 & \\
\hline $7 \times 7$ & 25.0591 & 16.0897 & 71.2220 & \\
\hline $13 \times 13$ & 23.5605 & 14.4160 & 68.9973 & \\
\hline & & & & \\
\hline & & & & \\
\hline
\end{tabular}

\section{CONCLUSIONS}

The input MRI brain tumor image is taken as the input and then added with speckle noise that would give noisy MRI brain tumor image. Noisy MRI brain tumor undergoes pre-processing stage, where wiener filtering is used. The parameters that are used in the wiener filter are the input image, type of the noise that is introduced to the MRI image and followed by mean and variance of the image. The pre-processing stage is followed by denoising stage, where the noisy MRI brain tumor is denoised using edge adaptive total variation technique. When the SNR value is high, the image resolution would be high when it is reconstructed. In the denoising stage different MRI brain tumor image is compared and SNR, peak SNR and MSE values are calculated. The denoising is followed by segmentation, where mean shift clustering and content based active contour segmentation techniques are used. The mean-shift algorithm with color and spatial information in color image segmentation is in generally successful for few cases. However, in some cases, the color and 
spatial information are not sufficient for superior segmentation. Mean Shift is a non- parametric clustering approach that does not take the form of the distribution and the number of clusters into consideration. Therefore, when used as a histological image segmentation method, mean shift can achieve better segmentation results than model based clustering systems. Finally, texture features are extracted from the clustered image. The Feature extraction that is used for extraction is gray level co-occurrence matrix algorithm, where contrast, correlation, energy and homogeneity is calculated. The extracted features are served as the input to classify the image. For improving the classification accuracy, the multiple features of the image and selection of a suitable classification methods are effectively used.

\section{FUTURE WORK}

In the future work the feature extraction will be followed by classification stage. Further the extracted data would be trained and then classified into either normal or abnormal brain based on the detection of presence of the tumor. The techniques used for classification are AVM, ANN, Hybrid techniques GA-ANN and GA-SVM, Deep learning with CNN. Finally, the results would be compared with these algorithms.

\section{REFERENCES}

[1] Ramesh Babu Vallabhaneni and V. Rajesh, "Brain Tumor Detection using Mean Shift Clustering and GLCM Features with Edge Adaptive Total Variation Denoising Technique", Alexandria Engineering Journal, Vol. 57, No. 4, pp. $2387-$ 2392, 2018.

[2] T. Chithambaram and K. Perumal, "Brain tumor Segmentation using Genetic Algorithm and ANN Techniques", Proceedings of IEEE International Conference on Power, Control, Signals and Instrumentation Engineering, pp. 970-982, 2017.

[3] T. Chithambaram and K. Perumal, "Brain Tumor Detection and Segmentation in MRI Images using Neural Network", International Journal of Advanced Research in Computer Science and Software Engineering, Vol. 7, No. 3, pp. 15041510, 2017.

[4] P. Chinmayi, L. Agilandeeswari, M. Prabu Kumar and K. Muralibabu, "An Efficient Deep Learning Neural Network based Brain Tumor Detection System", International Journal of Pure and Applied Mathematics, Vol. 117, No. 17, pp. 151-160, 2017.

[5] G. Malyadri, K.L. Sravani and Jyothi Kavathi, "Brain Tumor Detection System for Health Monitoring", International Journal of Pure and Applied Mathematics, Vol. 114, No. 10, pp. 103-108, 2017.

[6] P. Priyadarsni, B. Nandhini, A.R. Catherine, K. Sahana and K. Sundaravadivu, "Soft-Computing Assisted Tool to Extract Tumor Section from Brain MR Images", Proceedings of IEEE International Conference on Power, Control, Signals and Instrumentation Engineering, pp. 2776-2780, 2017.

[7] Megha Kadam and Avinash Dhole, "Brain Tumor Detection using GLCM with the Help of KSVM", International
Journal of Engineering and Technical Research, Vol. 7, No. 2, pp. 2454-4698, 2017.

[8] G.B. Praveen and Anita Agrawal, "Multistage Classification and Segmentation of Brain Tumor", IEEE International Conference on Computing for Sustainable Global Development, pp. 1628-1632, 2016.

[9] S. Shubhangi and Pradeep M. Patil, "Brain Tumor Classification using Artificial Neural Network on MRI Images", International Journal of Research in Engineering and Technology, Vol. 2, No. 12, pp. 218- 226, 2015.

[10] K. Machhale, H.B. Nandpuru, V. Kapur and L. Kosta, "MRI Brain Cancer Classification using Hybrid Classifier (SVMKNN)", Proceedings of International Conference on Industrial Instrumentation and Control, pp. 60-65, 2015.

[11] P. Sangeetha, "Brain Tumor Classification using PNN and Clustering", Proceedings of International Conference on Innovations in Engineering and Technology, Vol .3, No. 3, pp. 796-803, 2014.

[12] Snehal Basutkar, Aparna Davkhar, Bharat Mahajan and Moresh Mukhedkar, "Brain Tumor Detection using Segmentation", International Journal of Advance Engineering and Research Development, Vol. 3, No. 3, pp. 682-687, 2016.

[13] D. Haritha, "Brain Tumor Segmentation", International Journal of Advanced Technology in Engineering and Science, Vol. 4, No. 3, pp. 265-270, 2016.

[14] Shubhangi S. Veer and Pradeep M. Patil, "Brain Tumor Segmentation using GLCM", International Journal of Emerging Technologies and Engineering, Vol. 2, No. 9, pp. 131-135, 2015.

[15] Nameirakpam Dhanachandra, Khumanthem Manglem and Yambem JinaChanu, "Image Segmentation using K-means Clustering Algorithm and Subtractive Clustering Algorithm", Proceedings of $11^{\text {th }}$ International MultiConference on Information Processing, pp. 764-771, 2015.

[16] Shweta Jain, "Brain Cancer Classification using GLCM Based Feature Extraction in Artificial Neural Network", International Journal of Computer Science and Engineering Technology, Vol. 4, No. 7, pp. 966-970, 2013.

[17] S. Goswami and L.K.P. Bhaiya, "A Hybrid Neuro-Fuzzy Approach for Brain Abnormality Detection using GLCM Based Feature Extraction", Proceedings of International Conference on Emerging Trends in Communication, Control, Signal Processing and Computing Applications, pp. 1-7, 2013.

[18] A.R. Mathew, P.B. Anto and N.K. Thara, "Brain Tumor Segmentation and Classification using DWT, Gabour Wavelet and GLCM", Proceedings of International Conference on Intelligent Computing, Instrumentation and Control Technologies, pp. 1744-1750, 2017.

[19] W. Xu, X. Yue, Y. Chen and M. Reformat, "Ensemble of Active Contour-based Image Segmentation”, Proceedings of IEEE International Conference on Image Processing, pp. 86-90, 2017.

[20] Q. Sun and H. Tian, "Interactive Image Segmentation using Power Watershed and Active Contour Model", Proceedings of $3^{\text {rd }}$ IEEE International Conference on Network Infrastructure and Digital Content, pp. 401-405, 2012. 